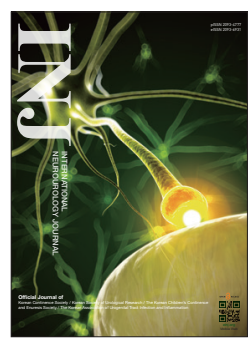

\title{
Role of 5'-AMP-Activated Protein Kinase Activators in Regulating Nuclear Factor Kappa B Signaling
}

\author{
Tack Lee (10) http://orcid.org/0000-0001-8508-9099 \\ Editor-in-Chief \\ Department of Urology, Inha University Hospital, Incheon, Korea \\ E-mail: lee.tack33@gmail.com
}

The role of nuclear factor kappa $\mathrm{B}(\mathrm{NF}-\mathrm{kB})$ is well known in immunity, inflammation, and cancer. Because NF- $\mathrm{kB}$ is associated with various diseases, such as arthritis, atherosclerosis, asthma, diabetes, and inflammatory bowel disease, many studies have focused on the I $\kappa B$ kinase (IKK)/NF- $\kappa B$ pathway as a treatment target [1]. In the bladder urothelium, NF- $\kappa B$ is associated with interstitial cystitis (IC) and bladder cancer. IC occurs as a result of inflammation and disruption of urothelium integrity. In addition, bladder inflammation induced by lipopolysaccharide (LPS) is mediated by the IKK/NF- $\mathrm{KB}$ pathway, and studies have shown that down regulation of NF- $\mathrm{KB}$, using anti-inflammatory drugs, can aid in treating such inflammation [2,3]. However, anti-inflammatory drug use aimed at reducing the symptoms of IC has yielded no significant results in true clinical practice.

LPS- and hypoxia-induced IKK-complex-mediated NF- $\mathrm{kB}$ phosphorylation of the human bladder cancer cell line was reduced by a $5^{\prime}$-AMP-activated protein kinase (AMPK) activator, 5 '-aminoimidazole-4-carboxamide ribonucleotide; therefore, AMPK activators may be one of the treatment drugs to regulate the IKK/NF- $\mathrm{KB}$ pathway [4]. AMPK presented close association with inflammation, and decreased AMPK activity is linked to increased inflammation induced by various types of insults [5]. Up-regulation of NF- $\kappa B$ signaling is one of underlying mechanisms associated with IC; therefore, AMPK activators may alleviate symptoms of IC by decreased NF- $\mathrm{kB}$ activity.

Various types of diseases presenting different characteristics sometimes share the same underlying pathophysiologic mechanism. The NF-kB signaling pathway plays a role in immune, inflammatory, and metabolic diseases as well as cancer. Moreover, such diseases are not caused by a single pathophysiology but instead, a complex of various mechanisms. Therefore, the pathophysiology of neurourology can often be understood through the findings from research on other human diseases, including cancer. Keeping an open mind regarding the mechanisms underlying various other diseases can improve the insight in the field of neurourology.

- Conflict of Interest: No potential conflict of interest relevant to this article was reported.

\section{REFERENCES}

1. Kumar A, Takada Y, Boriek AM, Aggarwal BB. Nuclear factor-kappaB: its role in health and disease. J Mol Med (Berl) 2004;82:434-48.

2. Wang XC, Saban R, Kaysen JH, Saban MR, Allen PL, Benes EN, et al. Nuclear factor kappa B mediates lipopolysaccharide-induced inflammation in the urinary bladder. J Urol 2000;163:993-8.

3. Velasco C, Angelico P, Guarneri L, Leonardi A, Clarke DE, Testa R. Effects of the nuclear factor-kappaB inhibitors 2-hydroxy-4-trifluoromethylbenzoic acid and aspirin on micturition in rats with normal and inflamed bladder. J Urol 2001;166:1962-8.

4. Choi BH, Lee DH, Kang JH, Park CS. Controls of nuclear factorkappa B signaling activity by $5^{\prime}$-AMP-activated protein kinase activation with examples in human bladder cancer cells. Int Neurourol J 2016;20:182-7.

5. Salminen A, Hyttinen JM, Kaarniranta K. AMP-activated protein kinase inhibits NF-kB signaling and inflammation: impact on healthspan and lifespan. J Mol Med (Berl) 2011;89:667-76. 